\title{
Erratum zu: Körperliche Aktivität und endotheliale Dysfunktion bei Typ-2-Diabetikern: über die Rolle von Stickstoffmonoxid und oxidativem Stress
}

\author{
Christian Brinkmann ${ }^{1}$, Robert H. G. Schwinger ${ }^{2}$ und Klara Brixius ${ }^{1}$ \\ ${ }^{1}$ Abteilung für Molekulare und Zelluläre Sportmedizin, Institut für Kreislaufforschung und Sportmedizin, \\ Deutsche Sporthochschule Köln, Köln, Deutschland \\ ${ }^{2}$ Medizinische Klinik II, Klinikum Weiden, Weiden i.d.Opf., Deutschland
}

Eingelangt am 15. September 2010, angenommen (nach Revision) am 22. Dezember 2010, online veröffentlicht am 7. March 2011

Erratum zu: Wien Med Wochenschr (2011) 161/11-12: 305-314

DOI 10.1007/s10354-011-0868-8

The second paragraph in the section entitled "Sportliche Aktivität beeinflusst den oxidativen Stress" (page 309) should read:

Begründen lässt sich das ROS-Aufkommen wahrscheinlich durch Beeinträchtigungen der mitochondrialen Atmung. Hierbei entweichen zunehmend Elektronen, und es werden Sauerstoffradikale gebildet. Desweiteren bringen vermutlich unter anderem folgende Abläufe durch akute Belastung vermehrt Radikale hervor: der verstärkte Abbau von Hypoxanthin im Xanthinoxidasesystem, die gesteigerte Autoxidation von Oxyhämoglobin, Oxymyoglobin sowie von Katecholaminen als auch die Infiltration von ROS-generierenden Leukozyten in das Muskelgewebe bei lokalen inflammatorischen Prozessen in Folge einer hohen mechanischen Beanspruchung der Muskulatur [58]. 